\title{
Archeology and evolution of QCD
}

\author{
A. De Rújula ${ }^{1,2, a}$ \\ ${ }^{1}$ CERN \\ ${ }^{2}$ IFT/UAM, Madrid
}

\begin{abstract}
These are excerpts from the closing talk at the "XIIth Conference on Quark Confinement and the Hadron Spectrum", which took place last Summer in Thessaloniki -an excellent place to enjoy an interest in archeology. A more complete personal view of the early days of QCD and the rest of the Standard Model is given in [1]. Here I discuss a few of the points which -to my judgement-illustrate well the QCD evolution (in time), both from a scientific and a sociological point of view.
\end{abstract}

\section{Introduction}

As one could judge from many talks at this conference [2], two areas of QCD have witnessed an enormous progress over the years. One of them is the non-perturbative first-principle lattice calculation of many relevant observables. The other embodies the next-to-next-to...leading-order results for many observed processes. How do some of these results compare with the corresponding earliest ones? How have the attitude and perceptions of "the community" evolved with time?

\section{Who invented quarks?}

The official history is that they were invented by Gell-Mann and Zweig, in that chronological order: Gell-Mann's published paper was received by Physics Letters on January 4th 1964, while Zweig's unpublished work is a CERN yellow report dated January 17th of the same year. But, as Napoleon is said to have said, History is the version of past events that people have decided to agree upon.

According to the same un-trustable source of the previous quote (Internet) Gell-Mann's paper was originally rejected by Physical Review Letters. Untrue [3]. None of this would significantly change the chronological order, which is anyway fairly irrelevant, the dates were so close. Concerning dates, it must be recalled that Gell-Mann wrote: These ideas were developed ... in March 1963; the author would like to thank Professor Robert Serber for stimulating them. For Serber's recollections, see [4].

A point in the official history [5] is lacking [1,6]. André Petermann published a paper (in French!) [7], received December 30th, 1963, shortly before the dates quoted in the first paragraph. In this paper he discusses mesons as made of a spinor/anti-spinor pair and baryons as composed of at least three spinors. Concerning the delicate issue of their charges, Petermann delightfully writes if one wants to preserve charge conservation, which is highly desirable, the spinors must have fractional charges. This fact is unpleasant, but cannot, after all, be excluded on physical grounds.

a e-mail: alvaro.derujula@cern.ch 
There are other unofficial issues concerning this chapter of the history of science. Was Zweig forbidden to have a preprint typed and to give a talk at CERN at the time? If so, by whom? I shall not answer these questions, but another one which I have been challenged to answer: why was the publication of Petermann's paper delayed for a year? Alas, nobody has found the original CERN preprint, yet. So, one cannot disprove something evil I have been told, namely that Petermann had plenty of time to change the paper before publication. That was not his style. Not only did he publish this paper in French -guaranteeing a dearth of readers- but in another uncontentious paper of his in the same journal, the editors clarify that the unusual delay in the publication was not the fault of the journal. The delay was seven years. The time needed by Petermann to bother correcting the proofs.

\section{Other quark mysteries, also unveiled}

The naive (constituent) quark model was impressively successful in its understanding of hadrons made of $u, d$ and $s$ quarks and in predicting the existence, decay pattern and mass of the $\Omega^{-}$[8]. But quarks and their confinement remained mysterious, more so because of the complementary evidence in SLAC's deep-inelastic electron-scattering experiments for charged constituents of protons [9], Feynman's partons [10], with "point-like" interactions with photons -Bjørken's scaling [11].

I shall not discuss the original literature on Yang-Mills theories [12], QCD [13], the electro-weak standard model [14-16], the necessary existence of charmed quarks [17] and the renormalizability of non-abelian gauge theories $[18,19]$. The discovery of strangeness-conserving neutral currents in neutrino scattering by the Gargamelle bubble-chamber collaboration at CERN [20] made experimentalists, and the world at large, aware of Yang-Mills theories, much as the 1971 work of 't Hooft [18] immediately attracted attention from (field) theorists to the same subject. For the hypothetical young reader I must emphasize that the fact that the Standard Model had all the chances of being "right" was at the time only obvious to and up-to-then overwhelmed minority of field-theory addicts [1].

The understanding of how quarks behaved when probed at short distances had to wait for the discovery ${ }^{1}$ of QCD's asymptotic freedom [21]. At the time David Politzer's office was next to mine at Harvard. David Gross and Frank Wilczek were at Princeton. The Harvard/Princeton competition was acute [22] and productive [1]. Suffice it to say that Harvard's motto is VERITAS (Truth), while Princeton's is DEI SVB NVMINE VIGET (God went to Princeton).

\section{A call for leniency}

Hereafter I am going to cite papers in an unbalanced way, with a large fraction of references to papers authored or coauthored by me. Part of this is a proximity effect, I am writing as a witness and a $1 / r^{2}$ law is inevitable, a price to pay for personal recollections, often more vivid than official histories.

As Golda Meyer put it: Don't be humble... you are not that great. That is correct in my case, but it does not apply to any of my to-be-cited coauthors, as the reader will easily recognize.

\section{$5 \alpha_{\mathrm{s}}$ and $\boldsymbol{\Lambda}_{\mathrm{QCD}}$}

In the mid 70's knives had been sharpened for long on inclusive reactions. The first concrete predictions of QCD [23] concerned the deviations from an exact scaling behaviour. But the available electron scattering and $e^{+} e^{-}$annihilation data [9] covered momentum transfers, $Q^{2}$, of not more than a few $\mathrm{GeV}^{2}$. Nobody (yet) dared to analyse these data in the "asymptotic" spirit of QCD. But some

\footnotetext{
${ }^{1}$ The remark, made also at this conference -that QCD's asymptotic freedom was first noticed by 't Hooft, Symanzik and the usual Russian suspects- made me italicize "discovery", which includes the realization of how important something may be.
} 
people not affected by dataphobia — a morbid condition of the brain (or brane?) that turns theoretical physicists into mathematicians- set out to exploit the only data then available at higher $Q^{2}$.

By the early '70s, the proton's magnetic form factor $G_{M}$ had been measured [24] up to $Q^{2} \sim 20$ $\mathrm{GeV}^{2}$. To bridge the gap between the QCD predictions for deep inelastic scattering and an elastic form factor, two groups [25, 26] used (or, with the benefit of hindsight, slightly abused) the then-mysterious "Bloom-Gilman duality" [27] relating the deep "scaling" data to the elastic and quasi-elastic peaks.

Not atypical of the Harvard/Princeton competition of those times, the papers I just quoted [25, 26] were received by the publisher on consecutive days. I prefer the one beginning: "Two virtues of asymptotically free gauge theories of the strong interactions are that they are not free-field theories and they make predictions that are not asymptotic"; to conclude "The results agree with experiment but are not a conclusive test of asymptotic freedom." The first statement was -then- not so obvious.

Being a bit more inclined to data analysis than my Princeton competitors -and wanting to be the first theoretical physicist [ (-: ] to measure a fundamental constant of nature- I extracted a value and an error range for $\Lambda_{\mathrm{QCD}}$, while David Gross and Sam Treiman simply chose a "right" value... somewhat surprisingly since their results — based on an analysis slightly different from mine — neither fitted the data nor subtracted from their confidence in the theory [26].

In figure 1 I show recent and very sophisticated results [28] on QCD's fine-structure "constant", $\alpha_{s}\left(Q^{2}\right)$, as well as my original leading-order result, $\alpha_{s}=12 /\left[25 \pi \log \left(Q^{2} / \Lambda^{2}\right)\right]$, and its error range. The vertical green double arrow shows, tongue in cheek, that the central value of the original determination of $\alpha_{s}$ must be reduced by $\sim 33 \%$ to get close to the current results.

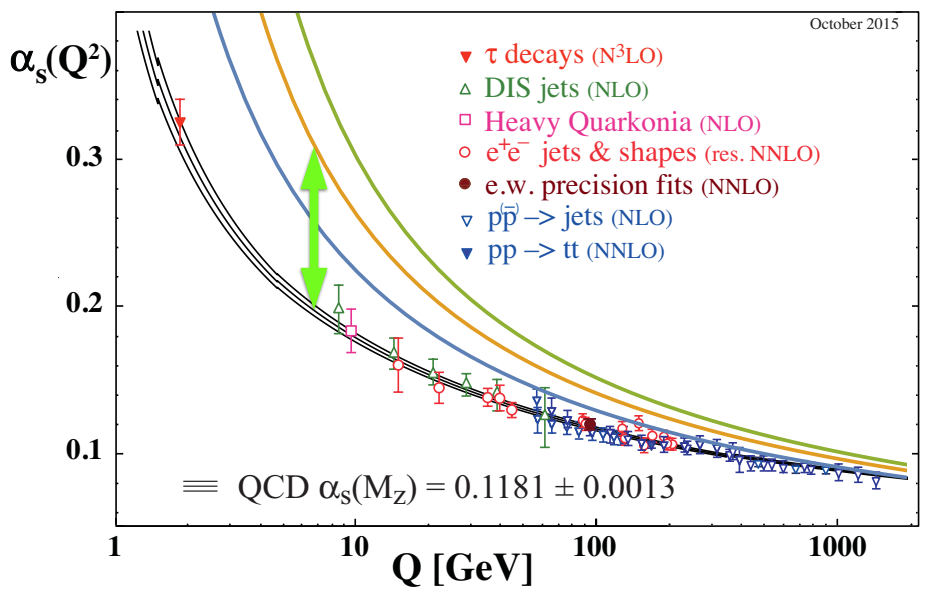

Figure 1. Recent (black lines) and early (colored lines) results on $\alpha_{s}\left(Q^{2}\right)$. From [28] and [25], respectively.

\section{Bloom-Gilman duality}

I was asked at this conference whether Bloom-Gilman duality (BGD) is a prediction of QCD. It is not (yet). That would require a complete understanding of bound-state production. But perturbative QCD explains BGD, in its QCD-improved realization. If you trust me, do not read this technical section.

BGD is the observation [27] that at low $Q^{2}$ a structure function shows prominent nucleon resonances, which "average" to the "scaling" function measured at some higher $Q_{0}^{2}$, and slide down its slope as $Q^{2}$ increases. As shown in figure 2, this happens if the chosen scaling variable in not Bjørken's $x=Q^{2} /\left(2 m_{p} v\right)$, but contains a "target mass correction", $1 / \omega^{\prime} \equiv x^{\prime}=Q^{2} /\left(2 m_{p} v+m_{p}^{2}\right)$. All this was considered at the time, in Californian style, as mystifying as a myth. 

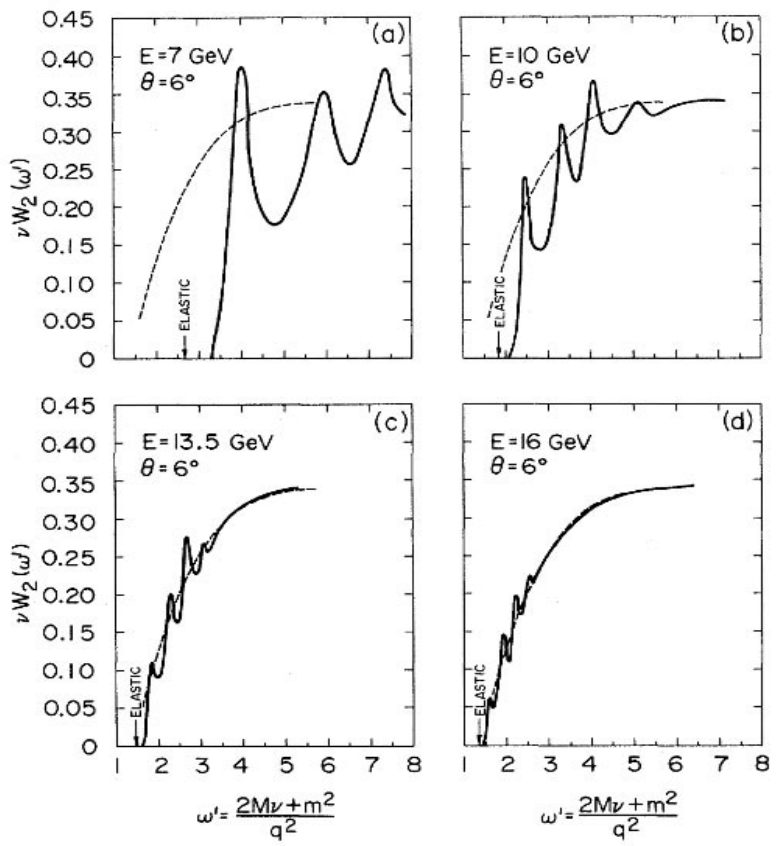

Figure 2. Bloom-Gilman duality at a fixed $e$-scattering angle and varying energies (or $Q^{2}$ values).

In Demythification of Electroproduction Local Duality and Precocious Scaling [29] Howard Georgi, Politzer and I argued that BGD is a consequence of QCD, inevitable if scaling is "precocious", as it must be for small $\Lambda$ (a fraction of a GeV). The scaling variable to be used, as we insisted in [30], is not $x^{\prime}$, but the one implied by a full use of QCD's operator-product expansion, i.e. Nachtman's variable $\xi=2 x /\left[1+\left(1+4 m_{p}^{2} x^{2} / Q^{2}\right)^{1 / 2}\right]$ [31], which takes care of the target-mass "higher-twist" effects of leading order $m_{p}^{2} / Q^{2}$. In studying the $Q^{2}$-evolution of the $n$-th moment of a structure function, weights $\xi^{n}$ and not $x^{n}$ ought to be used. The entire structure function is to be $\xi^{n}$-weighed, including the elastic contribution at $\xi=\xi_{p} \equiv 2 /\left[1+\left(1+4 m_{p}^{2} / Q^{2}\right)^{1 / 2}\right]$. The QCD duality is shown in figure 3 .

The crucial point is that the customary logarithmic QCD evolution of structure functions has higher-twist corrections. The next to leading-twist ones are of the form $\left(1+n a_{n} \Lambda^{2} / Q^{2}\right)$, with $\left|a_{n}\right| \simeq 1$, as the data allowed us to check in [29]. Consider taking the $n$-th $\xi$-moment of a structure function measured at a relatively large $Q_{0}^{2}$, where the resonant peaks are barely observable. Next, evolve this moment perturbatively down to a lower $Q^{2} \ll Q_{0}^{2}$. Since the $a_{n}$ are not perturbatively calculable, the perturbative prediction for the moment at the scale $Q^{2}$ has a relative uncertainty of $O\left(n \Lambda^{2} / Q^{2}\right)$.

To extract information on local duality from $n$ available moments, consider a polynomial $P_{n}(\xi)=$ $\sum_{0}^{n} C_{m} \xi^{m}$. One can find, for a given $n$, the $C_{m}$ 's corresponding to a best fit to a "window function" that can be used to test duality "locally", i.e. in a chosen interval $\xi_{1}$ to $\xi_{2}$, see figure 4. Given a predicted set of moments with uncertainties of $O\left(n \Lambda^{2} / Q^{2}\right)$ one expects a more local and precise QCD duality the smaller $n / Q^{2}$ is. That is precisely what is observed [29]. QED ${ }^{2}$.

The preceding detailed discussion justifies a posteriori the analysis of [25], based on BG local duality in an interval enclosing the elastic proton contribution $\propto G_{M}^{2} \delta\left(\xi-\xi_{p}\right)$. It also explains why those earliest attempts at QCD phenomenology resulted in a reasonable value for $\Lambda$.

\footnotetext{
${ }^{2}$ The actual analysis of QCD duality is a bit more elaborate, since one expects slightly different precisions for window functions centered at different $\xi$ 's [29].
} 

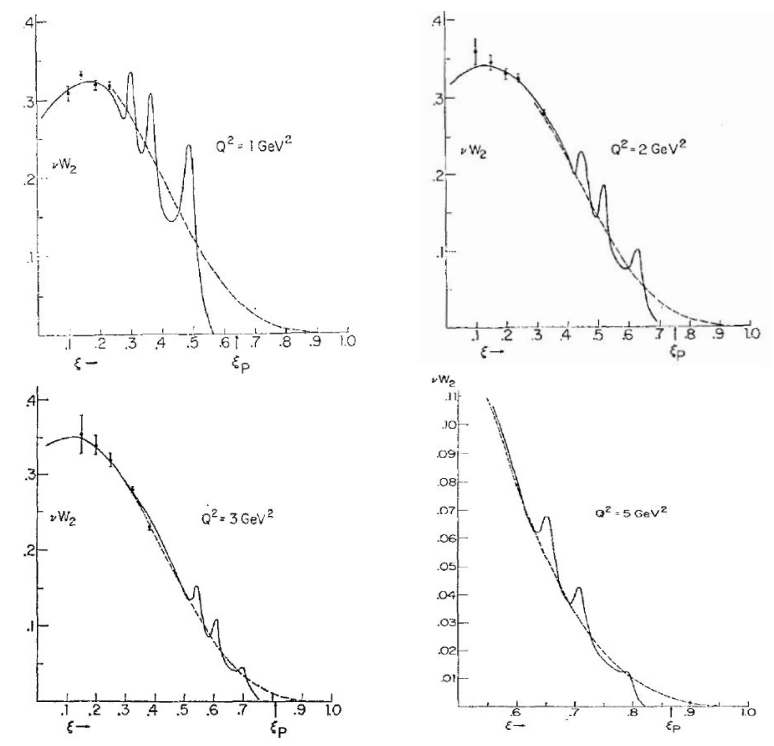

Figure 3. Dashed line: the perturbatively evolved proton structure function $v W_{2}\left(\xi, Q^{2}\right)$. Continuous line: a fit to actual data. $\xi_{p}$ is the position of the elastic contribution $\propto G_{M}^{2} \delta\left(\xi-\xi_{p}\right)$. A few data points are also shown.

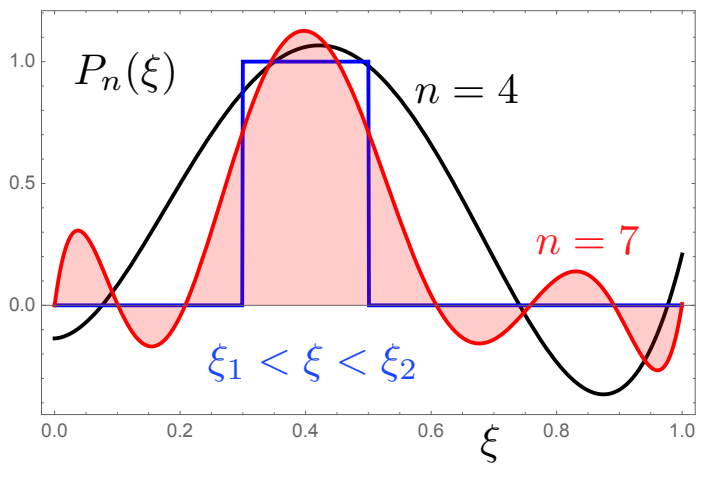

Figure 4. Best fits to a window function from $\xi_{1}$ to $\xi_{2}$ with polynomials $P_{n}(\xi)$, for $n=4$ and 7 .

\section{The irony of scaling in neutrino scattering}

The consensus that the observed scaling deviations smelled of QCD was not triggered by theorists, but by an analysis of neutrino data by Don Perkins et al. [32]. This test of QCD was not very severe. One reason is the neglect of higher twists [33]. Furthermore it is not possible, event by event, to measure the neutrino energy. Thus, in an unintended implementation of Bloom-Gilman duality, a measured structure function, $F_{v}\left(x, Q^{2}\right)$, is significantly blurred in $x$ and $Q^{2}$. This erases, at the low $Q^{2}$ of a good fraction of the data, the resonance bumps that must be there, as in figure 3. Moreover, the total $v$ cross section increased linearly with $E_{v}$ and was related to the naive, constituent-quark expectation from electroproduction by a famous $18 / 5$ factor, relating weak to electromagnetic quark charges.

Had the energy resolution of neutrino experiments been as good as that of their electron-scattering counterparts, the cross section rise would not have been so linear, the nucleon-resonance bumps in the structure functions would have been visible, and the data analysis would have had to be quite different. With a pinch of poetic license one could assert that, early on, many concluded that QCD was quite precise, but only because the data were not.

Elaborating on work by Giorgio Parisi [34], Georgi, Politzer and I explicitly worked out the $Q^{2}$ evolution of structure functions at fixed $x$, large $Q^{2}$ (for which $\xi \simeq x$ ) [35]. The simplest results are for 
$x F_{3}(x, Q)$ (the C-odd neutrino scattering structure function), shown in figure 5. A recent compilation of theory and HERA data for the electron scattering $F_{2}\left(x, Q^{2}\right)$ is also shown in the same figure.
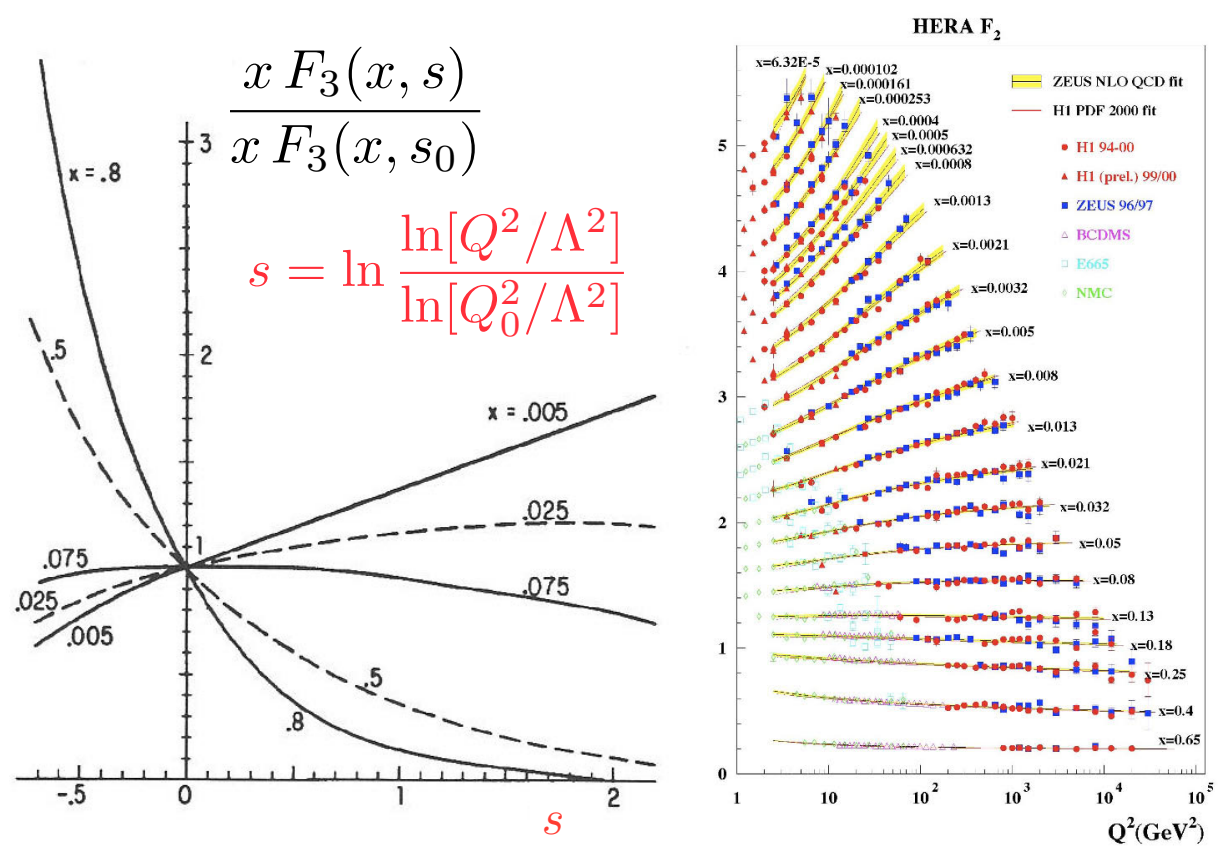

Figure 5. Left: Evolution of a normalized $v$ structure function $F_{3}(x, s) / F_{3}(x, 0)$ at fixed $x$. The trend has been corroborated in detail by a multitude of experiments (and theorists). Right: HERA electron-scattering data.

The results shown in the left panel of figure 5 were to become heavily used... and systematically referenced to authors of later papers. While yowling, I plead guilty to having learned much later that the simple underlying physics had been understood elsewhere: the renormalization-group [36, 37] picture of seeing partons within partons was drawn by Kogut and Susskind [38], the "physical gauge" diagrammatic image of a parton dissociating into others is due to Lev Lipatov [39], and its vintage QED analogue is none else than the Weiszäker-Williams equivalent-photon approximation [40].

\section{The November Revolution}

In the 1970's the pace of discovery was so fast that (lazy) journalists decided to prepare a "matrix article", wherein only the details of each specific discovery had to be filled in at the last minute. The NYT matrix article and its filling in November 1974 are shown in figure 6.

Ten days of November 1974 shook the world of physics. Something wonderful and almost [41] unexpected was to see the light of day: a very discreetly charmed particle [42, 43], a hadron so novel that it hardly looked like one. Thirty years later, it is not easy to recall the collective "high" in which this discovery, and others to be made in the two consecutive years, submerged the particle-physics community. In my opinion, a detailed account that reflects well the mood of the period is that by Riordan [44]. In a nutshell, the standard model arose from the ashes of the November Revolution, while its competitors died honourably on the battleground. 


\section{SCIENTIFIC DISCOVERY MA'TRIX AR'TICIE}

Recently, it was announced at press conferences that Prof. Ting and Prof. Richter and collaborators have stablished the existence of a new Particle

This is an important discovery corroborating the theory of Prof. Glashow and collaborators, who had predicted the existence of such a Particle as a consequence of the existence of Charmed Quarks

Other schools of thought interpret the new Particle as a manifestation of the long sought Whatchamacallit

$$
\begin{aligned}
& \text { Zellow entries filled by newspapers } \\
& \text { on November 6th and 7th, } 1974
\end{aligned}
$$

Figure 6. The Scientific Discovery Matrix Article. The November 74 Revolution; fully filled version.

A couple of survivors and many of the casualties are shown in figure 7. All of them but the last two were published, unrefereed, in the same issue of PRL. The comparison of the $J / \psi$ and its interpretations to the ones concerning the recent (un)discovery of the $750 \mathrm{GeV}$ state is... finding the proper adjective is left as an exercise for the reader, or for the hoards of referees.

Are the New Particles Baryon-Antibaryon Nuclei?

Alfred S. Goldhaber and Maurice Goldhaber

Interpretation of a Narrow Resonance in e+ e- Annihilation

Julian Schwinger

P Possible Explanation of the New Resonance in e+ e- Annihilation

R S. Borchardt, V.S. Mathur, and S. Okubo

Model with Three Charmed Quarks R. Michael Barnett

Heavy Quarks and e+ e- Annihilation Thomas Appelquist and H. David

Politzer

Is Bound Charm Found? A. De Rújula and S. L. Glashow

Figure 7. Immediate interpretations of the $J / \psi$, with their titles. PRL is Phys. Rev. Lett. 34, Jan. 6th, 1975. The last two papers are in Lett. Nuovo Cim. [45, 46]

Possible Interactions of the $\mathrm{J}$ Particle

H. T. Nieh, Tai Tsun Wu, and Chen Ning Yang

Is the 3104-MeV Vector Meson the psi - Charm or the W0?

G. Altarelli, N. Cabibbo, R. Petronzio, L. Maiani, G. Parisi

Charm, EVDM and Narrow Resonances in $e^{+} e^{-}$Annihilation

C. A. Dominguez and M. Greco

In the early Fall of '74, Tom Appelquist and David Politzer had been looking leisurely at how asymptotic freedom could imply a positronium-like structure for the $c \bar{c}$ bound states of a charmed quark and its anti-goodie-bean. In those days, both QCD and charm were already fully "established" at Harvard. Since Americans are often short of vocabulary, my first contribution to the subject was to baptize their toy charmonium. David and Tom's first charmonium spectrum was so full of Coulomblike peaks that they could not believe it themselves. They debated the problem long enough for the experimental avalanche to catch up with them. It was a heavy price to pay for probity. 
For an object of its mass, the $J / \psi$ is four orders of magnitude narrower than a conventional hadron resonance, and one order of magnitude wider than a then hypothetical weak intermediary. It could not be either. Of the multitude of theoretical papers of figure 7, only two attributed the narrow width to asymptotic freedom, one by Tom and David [41], who had intuited the whole thing before, the other one by Sheldon Glashow and me [47]. I recall Shelly storming the Lyman/Jefferson lab corridors with the notion of the feeble three-gluon hadronic decay of the $J^{P}=1^{-}$orthocharmonium state, and I remember Tom and David muttering: "Yeah". Our paper still made it to the publishers in the auspicious November, but only on the $27^{\text {th }}$, a whole week after the article of our Harvard friends. The paper by Cesareo Dominguez and Mario Greco also singled out charmonium as the interpretation.

We did a lot in our extra week [47]. Abusus non tollit usum (of asymptotic freedom) we related the hadronic width of the $J / \psi$ to that of $\phi \rightarrow 3 \pi$, to explain why this hadronic resonance had to be so narrow. We correctly estimated the yields of production of truly charmed particles in $e^{+} e^{-}$ annihilation, $v$-induced reactions, hadron collisions and photoproduction. Our mass for the $D^{*}$ turned out to be $2.5 \%$ off, sorry about that. We even discussed mass splittings within multiplets of the same quark constituency as hyperfine, a fertile notion. In discussing paracharmonium $\left(J^{P}=0^{-}\right)$we asserted that "the search for monochromatic $\gamma$ 's should prove rewarding". Finally, we predicted the existence of $\psi^{\prime}$, but this time it was our turn to be overtaken by the pace of discovery.

\section{Charmonium spectroscopy}

I have a few vivid printable recollections of the times I am discussing. One concerns the late night in which the existence of $P$-wave charmonia hit my head: we had been talking about $L=0$ states without realizing (we idiots!) that a bunch of $L=1$ charmonia should lie between $J$ and $\psi^{\prime}$ in mass. Too late to call Shelly, I spent hours guessing masses and estimating the obviously all-important $\gamma$-ray transition rates. At a gentlemanly morning hour I rushed on my bicycle to Shelly's office, literally all the way in, and attempted to snow him with my findings. I was speachless, out of breath and wits. Shelly profited to say: "I know exactly what you are trying to tell me, there are all these $P$-wave states etc., etc." He had figured it all out at breakfast. I hated the guy's guts.

In no time, David and Tom gathered forces with Shelly and me to produce an article [48] on Charmonium spectroscopy. Physical Review Letters was fighting its usual losing battle against progress (in nomenclature, $\smile$ ) and did not accept the title. Neither did PRL accept a similar title by our Cornell competitors [49]. The predicted spectra and the current experimental situation are shown in figure 8.

We, the Crimson, estimated the energy levels as "half-way" between those of a Coulomb and a harmonic oscillator potential. Indeed, a linear potential -adequate for confined $c \bar{c}$ states- is somewhat half-way. Our Cornell friends, the Carnelian, borrowed a linear-potential program from Ken Wilson and got similar results. Except for the all-important $\gamma$-ray transition rates, for which the Carnelian predictions were much better than ours.

\section{Hadron masses in a gauge theory}

Early in 1975, Georgi, Glashow, and I wrote a paper [50] whose style reflects how high we rode, as well as how unorthodox QCD still was. But for the added parentheses, here is how it began:

Once upon a time, there was a controversy in particle physics. There were some physicists who denied the existence of structures more elementary than hadrons, and searched for a self-consistent interpretation wherein all hadron states, stable or resonant, were equally elementary (the bootstrap). Others, appalled by the teeming democracy of hadrons, insisted on the existence of a small number of fundamental constituents (quarks) and a simple underlying force law (QCD). In terms of these more 

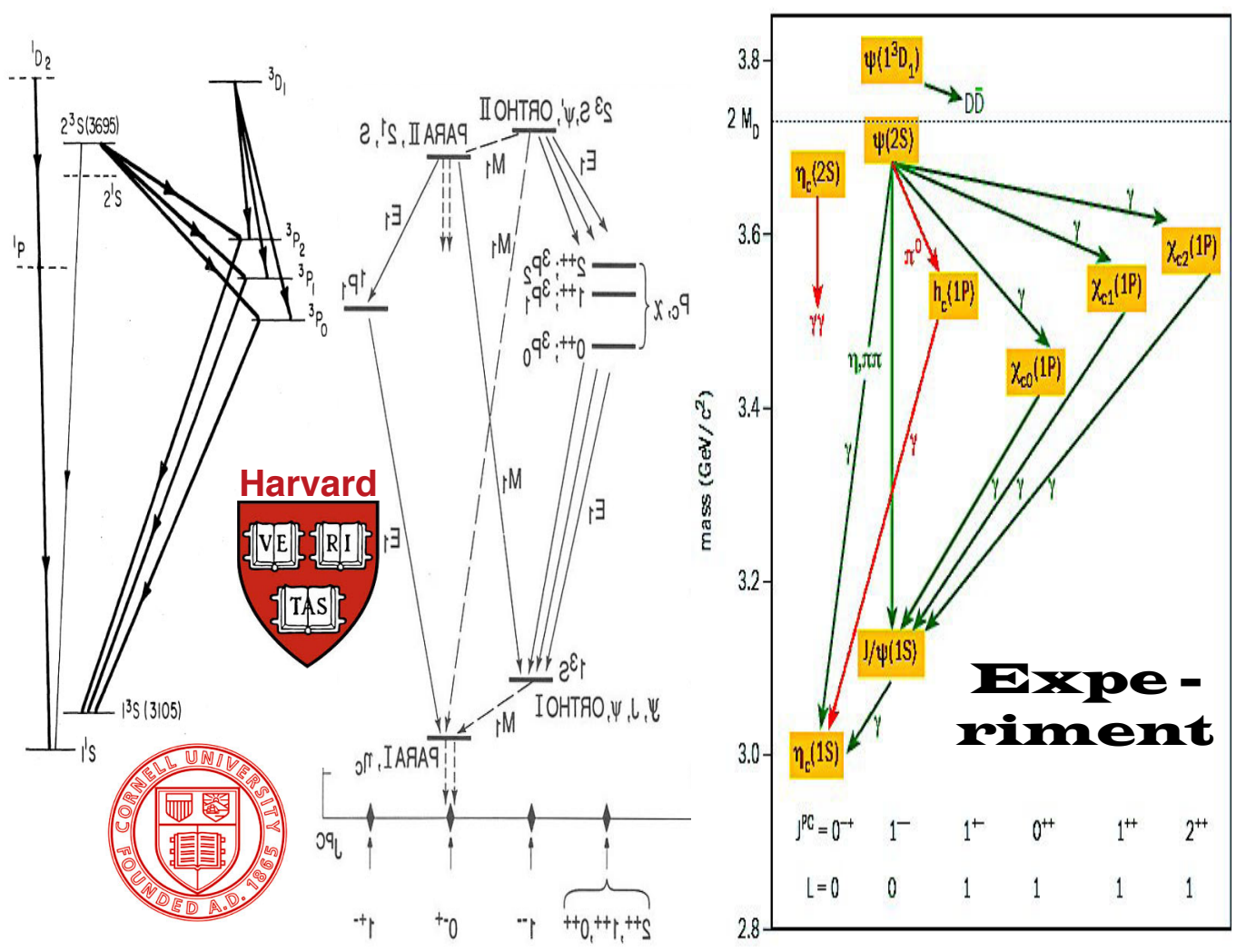

Figure 8. The spectra of charmonia. From left to right: Cornell's (squeezed), Harvard's (mirror reflected) and observed (with the inclusion of some non-radiative decays).

fundamental things, hadron spectroscopy should be qualitatively described and essentially understood just as are atomic and nuclear physics.

To the non-relativistic quark model, we added chromodynamic interactions entirely analogous to their electrodynamic counterparts. We shall see that to this day it is not totally clear why the ensuing predictions were so good. Our paradigmatic result was the explanation of the origin and magnitude of the $\Sigma^{0}-\Lambda$ mass difference. The two particles have the same spin and quark constituency, their mass difference is a hyperfine splitting induced by spin-spin interactions between the constituent quarks. A little later, the "MIT bag" community published their relativistic version [51] of the same work.

In [50] we also predicted the masses of all ground-state charmed mesons and baryons and (me too, I'm getting bored with this) we got them right on the mark. Predictions based on an incredible SU(4) version of the Gell-Mann-Okubo SU(3) mass formula, and also the more sensible bag results, turned out to be wrong. Only one person -indeed, again a Russian- trusted a "QCD-improved" constituent quark model early enough to make predictions somewhat akin to ours: Andrei Sakharov [52].

\subsection{Good News at Last}

While theorists faithfully ground out the phenomenology of QCD, experimentalists persistently failed to find decisive signatures of our Trojan horse: the charmed quark. At one point, the upper limits 
on the $\gamma$-ray transitions of charmonia were well below the theoretical expectations. Half of the $e e$ cross-section above $\sqrt{s}=4 \mathrm{GeV}$ was due to charm production, said we. Who would believe that experimentalists couldn't tell?

In the winter of '75 we saw a lone ray of light. As Nick Samios recalls in detail in [53], a Brookhaven bubble-chamber group [54] pictured a $\Delta S=-\Delta Q$ event, forbidden in a charmless world, and compatible with the chain:

$$
v_{\mu} p \rightarrow \Sigma_{c}^{++} \mu^{-} ; \quad \Sigma_{c}^{++} \rightarrow \Lambda_{c}^{+} \pi^{+} ; \quad \Lambda_{c}^{+} \rightarrow \Lambda \pi^{+} \pi^{+} \pi^{-}
$$

Two charmed baryons discovered in one shot! This was a source of delight not only for us, but also for the experimentalists involved. They deserve a reproduction of their event: figure 9 , and a quotation:
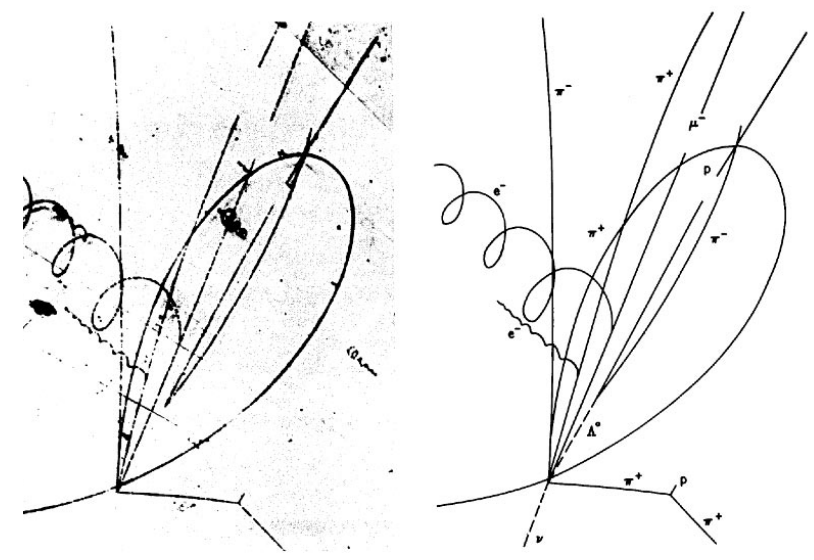

Figure 9. The Brookhaven doubly charming event [54].

The total recoiling hadron mass $\left(\Lambda \pi^{+} \pi^{+} \pi^{-}\right)$[is] $2426 \pm 12 \mathrm{MeV}$.

This mass is in reasonable agreement with the value predicted by De Rújula, Georgi and Glashow for the lowest-lying charmed-baryon states of charge $+2,2420 \mathrm{MeV}\left(\mathrm{J}^{P}=\frac{3}{2}^{+}, I=1, \Sigma_{c}^{*}\right) \ldots$ There are three $\pi^{+}$'s and thus three mass differences derivable form this event; these are observed to be $166 \pm 15$ $\mathrm{MeV}, 338 \pm 12 \mathrm{MeV}$, and $327 \pm 12 \mathrm{MeV}$. The first of these differences is in remarkable agreement with the $160 \mathrm{MeV}$ predicted for the decay of a spin- $\frac{1}{2}$ charmed baryon $\Sigma_{c}$ decaying into a charmed $\Lambda_{c}$.

This is almost precisely the way I feel experimentalists should write papers. Only "almost" because the agreement between $2426 \pm 12$ and $2420 \mathrm{MeV}$ seems to me to be a bit better than "reasonable".

\subsection{Back to the future}

By now the masses of many mesons and baryons have been precisely post-dicted in lattice QCD. It is perhaps instructive to look at an instance: charmed baryons. This is done in figure 10, where a collection of lattice results [55] is shown, along with the observed values (blue lines) for the positive parity baryons we are concerned with. Also shown in the figure are the predictions of [50], made after the discovery of the $J / \psi$, but before that of open charm. This limited information made us (over)estimate a common uncertainty of $\pm 50 \mathrm{MeV}$-reflected as the ellipses- around the central values.

The QCD-improved naive quark model predictions of [50] still compete with the lattice results. The moral is that there is an element of "truth" in the naive model, or in quenched lattice results. Presumably, if latticists could device a gauge-invariant way to characterize a confined constituentquark propagator, it would be found to peak at a "constituent mass" some $300 \mathrm{MeV}$ larger than a 


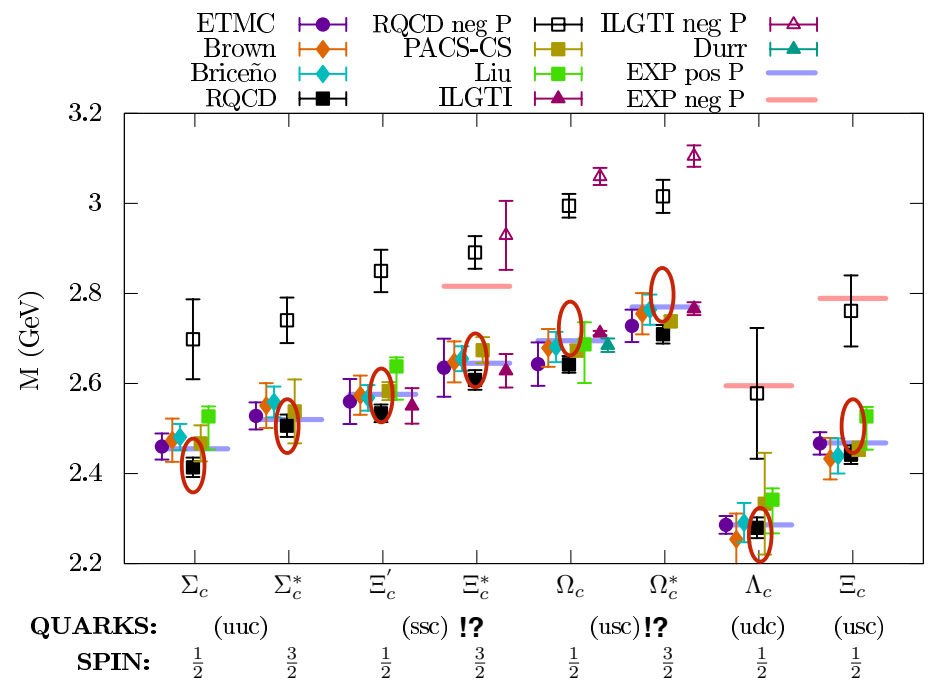

Figure 10. Charmed baryons. Blue traits: mass measurements for the positive parity ones. Lattice results compiled in [55] [(ssc) and (usc) are interchanged]. Ellipses: the predictions of [50], with their common uncertainty.

"Lagrangian" or chiral-model mass ${ }^{3}$. Similarly, they might discover one gluon exchange dominance for the mass differences between baryons of the same quark constituency but different spin.

\subsection{Beyond $q \bar{q}$ and qqq}

A currently very active endeavor is the analysis of hadrons with a larger quark constituency than the consuetudinary old one ${ }^{4}$. The theoretical prehistory of this subject dates back to the mid 70's [57]. Perhaps the first data analyses with Molecular Charmonium in mind were those of [58, 59]. In the first

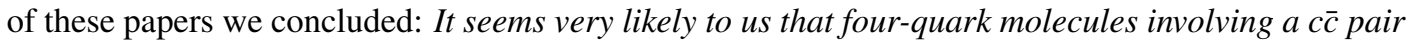
do exist, and have a rich spectroscopy. Our conjecture that the $4.028 \mathrm{GeV}$ and perhaps de $4.4 \mathrm{GeV}$ peaks in $e^{+} e^{-}$annihilation are indeed due to the production of these molecules is more speculative. If it is true, then nature has provided us with a spigot to a fascinating and otherwise almost inaccessible new "molecular" spectroscopy full of experimental and theoretical challenges.

This conclusion is still unaltered, but for two details: our lack of prescience in the almost inaccessible stipulation, and the possibility that some of the new hadrons are more "nuclear" than "molecular". That the situation would be very messy, even in a narrow energy domain in $e^{+} e^{-}$annihilation, could already be concluded from figure 11. I have no idea whether any of its predictions are correct.

\section{Back to the past}

In the summer of ' 75 -after a year of upper limits incompatible with the theoretical expectationsevidence finally arose for the $P$-wave charmonia [60]. The DESY experimentalists did not refer to the theorists who suggested their search; they are hereby punished: they do not get a reference, and they will remain eternally ignorant of my juicy version of the story of their competition with SLAC.

The discovery of the positronium-like $c \bar{c}$ spectrum of figure 8 started to convert many infidels to the quarker faith. And the charmed quark, not yet found unaccompanied by its antiparticle, was to continue playing a crucial role in the development and general acceptance of the standard lore.

\footnotetext{
${ }^{3}$ This happens in Bethe-Saltpeter models of confinement [56]. I am indebted to Pilar Hernandez for input on this topic.

${ }^{4}$ See e.g. the talks by Marina Nielsen and Sebastian Neubert at this conference [2].
} 


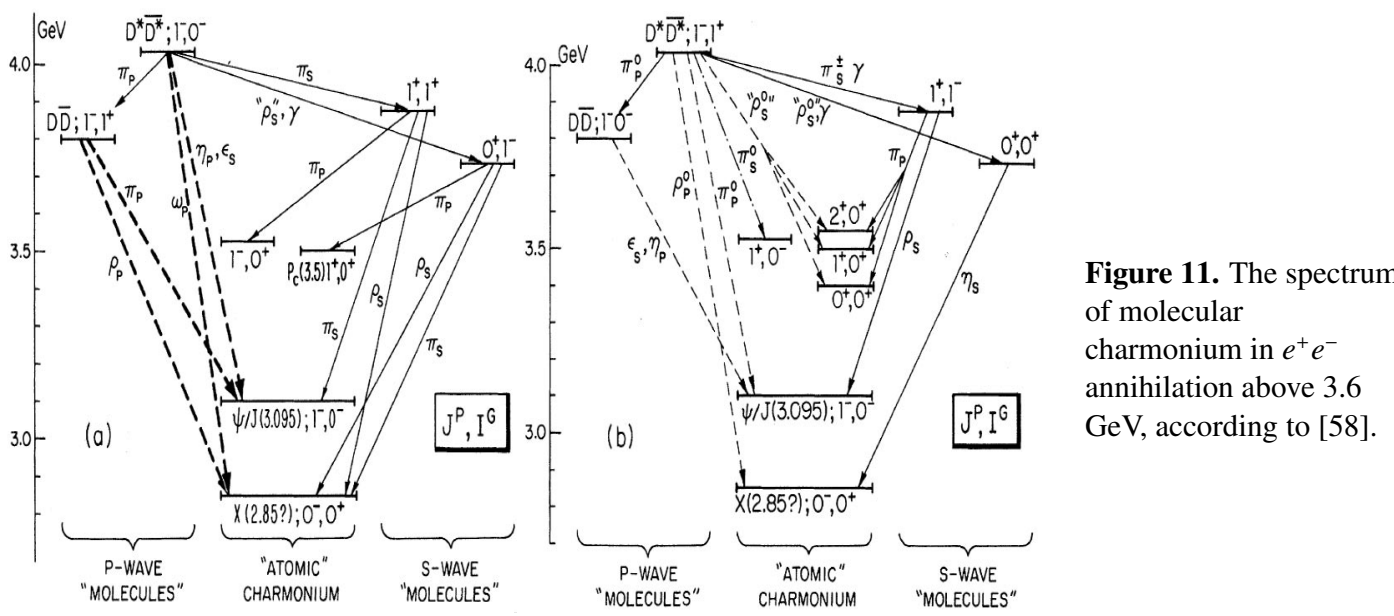

\section{Yet Another Year of Lank Cows}

As shown in figure 12, measurements at SLAC of the ratio $R \equiv \sigma\left(e^{+} e^{-} \rightarrow\right.$ hadrons $) / \sigma\left(e^{+} e^{-} \rightarrow \mu^{+} \mu^{-}\right)$ showed a doubling of the yield and structure aplenty as the $\sqrt{s} \sim 4 \mathrm{GeV}$ region is crossed [61]. Much of the jump had to be due to the production of charmed pairs, which were not found. Howard Georgi and I innocently believed that a serious sharpening of the arguments would help.

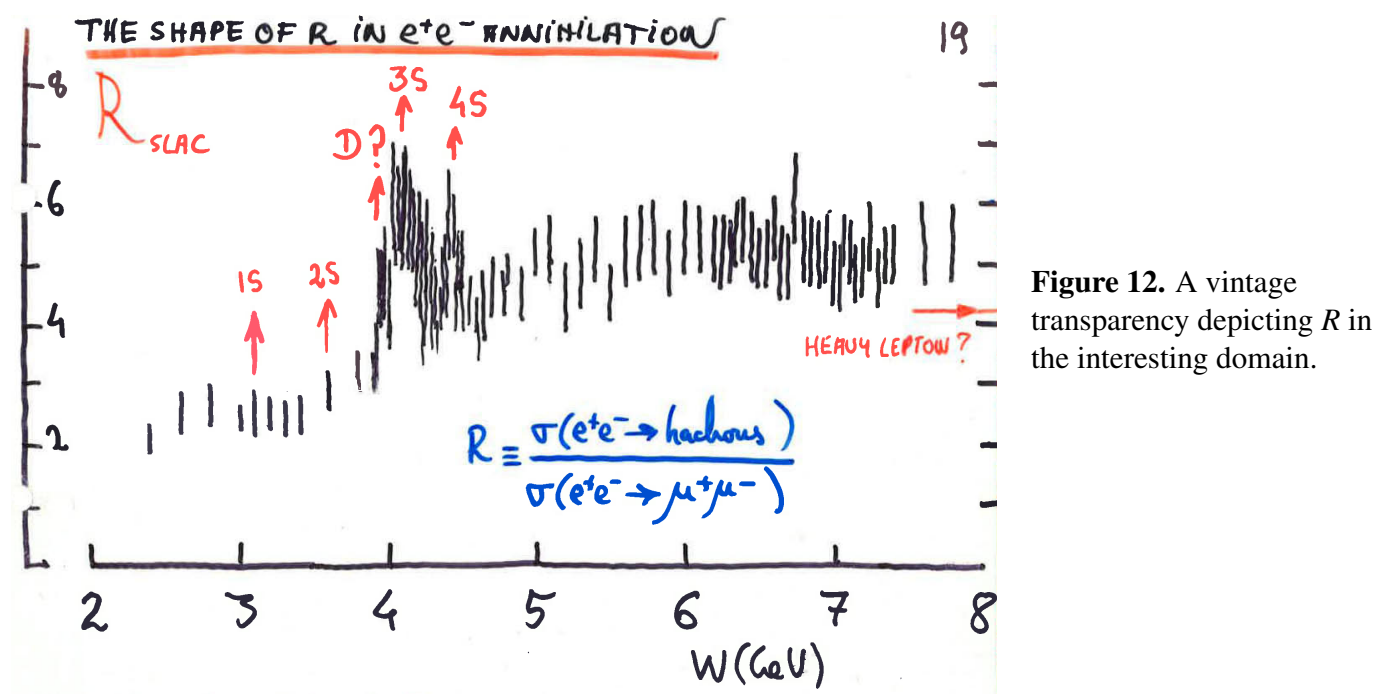

In the space-like domain, $s<0$, QCD predictions for $e^{+} e^{-}$annihilation are insensitive to thresholds, bound-state singularities and hadronization caveats. For years, theorists had been unjustifiably 
applying the predictions to the time-like domain wherein experimentalists insist on taking $e e$ data. In a paper [62] whose rhythmic title Finding Fancy Flavours Counting Colored Quarks was duly censored, we transferred the $e^{+} e^{-}$data, via a dispersion relation, to a theoretically safer space-like haven. This somersault [63] allowed us to conclude that the old theory with no charm is excluded, the standard model with charm is acceptable if heavy leptons are produced, and six quark models are viable if no heavy leptons are produced. Thus, anybody listening to the other voice in the desert (that of Martin Perl, who was busy demonstrating that he had discovered the $\tau$ ) had no choice but charm.

Our work was improved by Enrico Poggio, Helen Quinn and Steven Weinberg [64], who realized that one could, in the complex $s$-plane, work in a contour around the real axis where perturbative QCD can still be trusted, whilst the distance from the dirty details of real life is judged safe. The work of Enrico, Helen and Steve further strengthened our conclusion: the measured total cross section, analyzed on firm theoretical grounds, implied the existence of charm and of a new heavy lepton.

Imagine that some theorists, analyzing LHC data with the current Standard Model -with its six quarks and three charged leptons- and on the basis of a ship-shape analysis with a statistical evidence so strong that there was no need to count $\sigma$ 's, proved that an extra quark and an extra charged lepton were being produced. There is no doubt that the community would conclude that the cited theorists had discovered these particles. But the social power of preconceptions cannot be overestimated. Prior to 1976, the Standard Model -then having three established quarks and two observed charged leptonswas not yet accepted as "part of the truth". Thus, to be believed, the analyses in [62] and [64], had to wait for the explicit discovery of open charm and the $\tau$ lepton.

\section{Charm is found}

No amount of published information can compete with a few minutes of conversation. The story, whose moral that was, is well known. For the record, I should tell it once again $[1,65,66]$ :

Shelly Glashow happened to chat with Gerson Goldhaber in an airplane. Surprisingly, the East Coast theorist managed to convince the West Coast experimentalist of something. There was no way to understand the data unless charmed particles were being copiously produced above $\sqrt{s}=3.7 \mathrm{GeV}$. The experimentalists devised an improved (probabilistic) way to tell kaons from pions. In a record 18 days two complementary SLAC/LBL subgroups found convincing evidence for a new particle with all the earmarks of charm [67]. The charmonium advocates at Cornell had been trying for a long time to convince the experimentalists to attempt to discover charm by sitting on the $\psi(3440)$ resonance, or on what would become a "charm factory": $\psi(3770)$ [68]. Alas, they initially failed.

The observation of charmed mesons ought to have been the immediate happy ending, but there was a last-minute delay. The invariant-mass spectrum of recoiling stuff in $e^{+} e^{-} \rightarrow D^{0}\left(D^{ \pm}\right)+\ldots$ had a lot of intriguing structure, but no clear peak corresponding to $D^{0} \bar{D}^{0}$ [67] or $D^{+} D^{-}$[69] associated production. Enemies of the people rushed to the conclusion that what was being found was an awful mess, and not something as simple as charm, as in figure 13 .

But we had one last unspent cartridge [70]. We expected $D \bar{D}, D \bar{D}^{*}+\bar{D} D^{*}$, and $D^{*} \bar{D}^{*}$ production to occur in the "spin" ratio 1:4:7 (thus the $D \bar{D}$ suppression). We trusted our prediction [50] $m\left(D^{*}\right)-$ $m(D) \simeq m(\pi)$, which implies that for charm production close to threshold, the decay pions are slow and may be associated with the "wrong" $D$ or $D^{*}$ to produce fake peaks in recoiling mass. Finally,

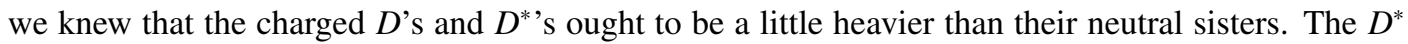
decays had to be very peculiar: $D^{* 0} \rightarrow D^{+} \pi^{-}$is forbidden, $D^{* 0} \rightarrow D^{0} \gamma$ competes with $D^{* 0} \rightarrow D^{0} \pi^{0}$, etc. On the basis of these considerations (and with only one fit parameter) we constructed the recoil spectra shown in figure 14. Case closed! 


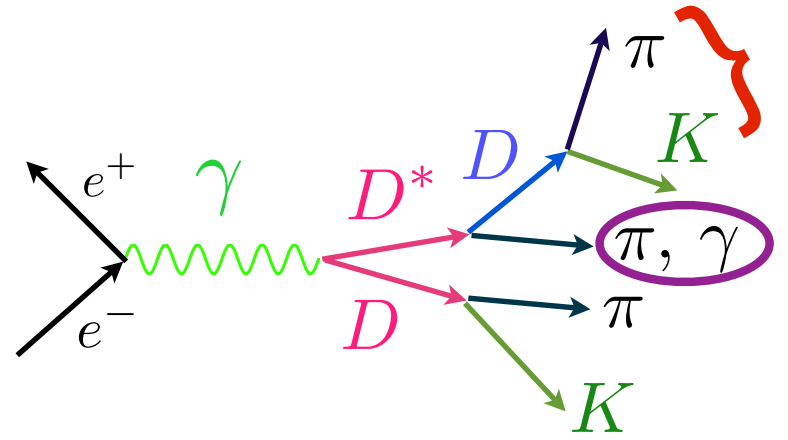

Figure 13. $\bar{D} D^{*}$ pair-production. Invariant masses (IM) are measured as recoiling from $K \pi$ (as in the figure), or $K \pi \pi$ ensembles. The decay $D^{*} \rightarrow D \pi$ is allowed, forbidden or suppressed, depending on the particle's charges [50]. Close to the open-charm threshold all observed hadrons are "slow" and fake IM peaks consequently occur.
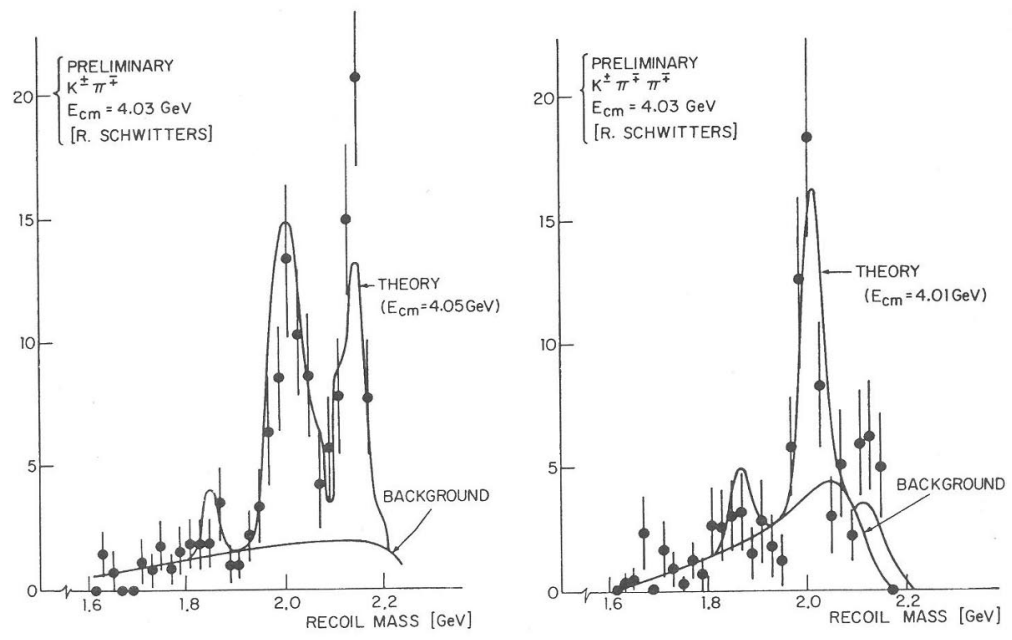

Figure 14. Predicted and observed invariant-mass spectra, recoiling against neutral and charged $D$ 's. The theoretical curves are a one-parameter description [70].

\section{Conclusion}

In the olden days experimentalists, particularly the ones working in the East Coast of the USA, were strongly motivated to disprove all theories and to mistrust almost all theorists; they were perhaps permeated by some arcane Californian faith that nature is intrinsically unfathomable. They definitely did not have in their data-analysis programs the current instruction stating: IF [RESULT = STANDARD; LOOK ELSEWHERE]. This made life most enjoyable and the case for the then-challenged standard model veeeery strong. To gauge whether or not things have changed, consider supersymmetry.

Even the most formal theorist or the most cable-connecting experimentalist understands positronium and hydrogen. These objects are not so very different from their QCD analogs, charmonium and charmed particles. This may be why it took asymptotic freedom and a fourth (charmed) quark to have the Standard Model become the standard lore. 


\section{Windup}

The most challenging QCD problem -confinement-is not yet solved, in spite of a one M\$ prize awaiting whoever solves it [71]. Factorization in deep inelastic scattering (the fact that one can use parton distribution functions) is proven [72]. But it is not proved for Drell-Yan processes, a crucial basis in the analyses of LHC data. Nowadays theorists are also considering processes allegedly initialized by multiple partons. This is even less solidly founded. And there is no prize for clarifying any of this.

Independently of the above caveats, as this conference reflected quite well, there has been an enormous progress in perturbative and non-perturbative QCD since quarks were invented, in 1963. This phenomenological progress often required phenomenally difficult theoretical developments, and played a key role in the understanding of experimental results, most recently at the LHC. It is difficult not to feel that particle physics phenomenology is generally less appreciated than it should, particularly in comparison with theoretical work "beyond" this or that. A reader who has reached this far is presumably a QCD phenomenologist. I have no doubt that (s)he would agree with me on all this.

Acknowledgments. I am most indebted to my collaborators, in particular the ones I have cited. In alphabetical order: Tom, Howard, Shelly and David. I am also thankful to Johannes Bluemlein, Cesareo Dominguez, Mario Greco, Marek Karliner and Martinus Veltman for comments and suggestions. This project has received funding from the European Union's Horizon 2020 research and innovation programme under the Marie Sklodowska-Curie grant agreement No 674896.

\section{References}

[1] A. De Rújula hep-ph/0404215, in Fifty years of gauge theories edited by: Gerardus 't Hooft (Utrecht University, The Netherlands). World Scientific Pub Co. pages 399-432

[2] https://indico.cern.ch/e/conf12

[3] http://www.webofstories.com/play/murray.gell-mann/115

[4] R. Serber \& R.B. Crease. Peace $\mathcal{G}$ War: Reminiscences of a Life on the Frontiers of Science, Columbia University Press. ISBN 978-0-231-10546-0, (1998) page 199

[5] Many personal recollections on the history of the Standard Model can be found in The Rise of the Standard Model: A History of Particle Physics from 1964 to 1979. Hoddeson et. al. editors. Cambridge Univ. Press (1997)

[6] A. De Rújula, CERN Courier, April 30, 2014; http://cerncourier.com/cws/article/cern/56949

[7] A. Peterman, Nuc. Phys. 63, 349, (1965)

[8] M. Gell-mann \& Y. Ne'eman, The eightfold way Advanced Book Classics, 2000.

[9] G. Miller et al., Phys. Rev. D5, 528 (1972)

[10] R.P. Feynman, Phys. Rev. Lett. 23, 1415 (1969)

[11] J.D. Bjørken, Phys. Rev. 179, 1547 (1969)

J.D. Bjørken \& E.A. Paschos, Phys. Rev. 158, 1975 (1969)

[12] C.-N. Yang \& R.L. Mills, Conservation of isotopic spin and isotopic gauge invariance ${ }^{5}$, Phys. Rev. 96, 191 (1954)

[13] M. Gell-Mann, H. Fritzsch \& J. Leutwyler, Advantages of the color-octet gluon picture, Phys. Lett. B47, 365 (1973)

[14] S.L. Glashow, Partial symmetries of weak interactions, Nucl. Phys. 22, 579 (1961)

[15] S. Weinberg, A model of leptons, Phys. Rev. Lett. 19, 1264 (1967)

\footnotetext{
${ }^{5}$ From here downwards I have taken the liberty of specifying some article's titles.
} 
[16] A. Salam \& J.C. Ward, Electromagnetic and weak interactions, Phys. Lett. 13, 168 (1964);

A. Salam, in Elementary Particle Theory; ed. N. Svartholm (Stockholm, 1968).

[17] S.L. Glashow, J. Iliopoulos \& L. Maiani, Phys. Rev. D2, 1285 (1970)

[18] G. 't Hooft, Renormalizable Lagrangians for massive gauge field theories, Nucl. Phys. 35, 167 (1971)

[19] G. 't Hooft \& M.J.G. Veltman, Regularization and renormalization of gauge fields, Nucl. Phys.

B44, 189 (1972)

[20] F.J. Hasert et al. Without muon, Phys. Lett. 46 B, 138 (1973)

[21] H.D. Politzer, Phys. Rev. Lett. 26, 1346 (1973)

D. Gross \& F. Wilczek, Phys. Rev. Lett. 26, 1343 (1973)

[22] H.D. Politzer, Nobel Lecture The dilemma of attribution, Rev. Mod. Phys. 77, 851 (2005)

[23] D.J. Gross \& F. Wilczek, Phys. Rev. D8, 3633 (1973), D9, 980 (1974)

T. Appelquist \& H. Georgi, Phys. Rev. D8, 4000 (1973)

A. Zee, Phys. Rev. D8, 4038 (1973)

H. Georgi \& H.D. Politzer, Phys. Rev. D9, 416 (1974)

[24] P.N. Kirk et al., Phys. Rev. D8, 63 (1973)

[25] A. De Rújula, Phys. Rev. Lett. 32, 1143 (1974)

[26] D.J. Gross \& S.B. Treiman, Phys. Rev. Lett. 32, 1145 (1974)

[27] E. Bloom \& F. Gilman, Phys. Rev. D4, 2901 (1971)

M. Nauenberg, Acta Phys. Hung. 31, 51 (1972)

[28] A. Pich at this conference

C. Patrignani et al. Particle Data Group, Chin. Phys. C40, 100001 (2016)

[29] A. De Rújula, H. Georgi \& H.D. Politzer, Ann. Phys. (N.Y.) 103, 315 (1977)

[30] A. De Rújula, H. Georgi \& H.D. Politzer, Phys. Lett. 64B, 428 (1976)

[31] O. Nachtmann, Nucl. Phys. B63, 237 (1973)

[32] P.C. Bosetti et al., Nucl. Phys. B142, 1 (1978)

[33] L.F. Abbott \& R.M. Barnet, Ann. Phys. 125, 276 (1980)

A. De Rújula, J. Ellis, R. Petronzio, G. Preparata \& W. Scott, Can one tell QCD from a hole in the ground? A drama in five acts in Pointlike structures inside and outside hadrons, The 1979 International School of Subnuclear Physics, Erice, Sicily.

[34] G. Parisi, Phys. Lett. 43B, 207 (1973)

[35] A. De Rújula, H. Georgi \& H.D. Politzer, Phys. Rev. D10, 2141 (1974)

[36] E. Stuekelberg \& A. Petermann, La normalisation des constantes dans la théorie des quanta, Helv. Phys. Acta 26, 499 (1953)

[37] M. Gell-Mann \& F. Low, Quantum electrodynamics at small distances, Phys. Rev. 95, 1300 (1954)

[38] J. Kogut \& L. Susskind, Phys. Rev. D9, 697 (1974)

[39] L.N. Lipatov, Sov. J. Nucl. Phys. (Yad. Phys.) 20, 181 (1974), and references therein.

[40] C. Weiszäker \& E.J. Williams, Z. Phys. 88, 612 (1934)

[41] T.W. Appelquist \& H.D. Politzer, Phys. Rev. Lett. 3443 (1975)

[42] J.J. Aubert et al., Phys. Rev. Lett. 33, 1404 (1974)

[43] J.-E. Augustin et al., Phys. Rev. Lett. 33, 1406 (1974)

[44] M. Riordan, The Hunting of the Quark, A True Story of Modern Physics. New York, USA: Simon \& Schuster (1987). (A Touchstone Book).

[45] G. Altarelli, N. Cabibbo, R. Petronzio, L. Maiani \& G. Parisi. Lett. Nuovo Cim. 11, 609 (1975) 
[46] C. A. Dominguez and M. Greco. Lett. Nuovo Cim. 12, 439 (1975)

[47] A. De Rújula \& S.L. Glashow, Phys. Rev. Lett. 34, 46 (1975)

[48] T.W. Appelquist, A. De Rújula, S.L. Glashow, \& H.D. Politzer, Phys. Rev. Lett. 34, 365 (1975)

[49] E. Eichten, K. Gottfried, T. Kinoshita, J. Kogut, K.D. Lane, \& T.-M Yan, Phys. Rev. Lett. 34, 369 (1975)

[50] A. De Rújula, H. Georgi \& S.L. Glashow, Phys. Rev. D12, 147 (1975)

[51] T. De Grand, R.L. Jaffe, K. Johnson \& J. Kiskis, Phys. Rev. D12, 2060 (1975)

[52] A.D. Sacharov, Pisma JETP 21, 554 (1975)

[53] N. Samios in History of Original Ideas and Basic Discoveries in Particle Physics, eds. H. B.

Newman \& T. Ypsilantis, Erice 1994, Plenum Press, New York, 1996.

[54] E.G. Cazzoli et al., Phys. Rev. Lett. 34, 1125 (1975)

[55] P. Perez-Rubio, S. Collins and G. S. Bali, Phys. Rev. D92, 034504 (2015)

[56] R.T. Cahill \& S.M. Gunner, Phys.Lett. B359, 281 (1995)

[57] L.B. Okun \& M.B. Voloshin, Pis'ma Zh. Eksp. Teor. Fiz. 23, 369 (1976)

R.L. Jaffe, unpublished

M. Bander et al. Phys.Rev.Lett. 36695 (1976)

C. Rosenweig, Phys.Rev.Lett. 36697 (1976)

Nee-Pong Chang and C.A. Nelson, unpublished.

[58] Molecular Charmonium: A New Spectroscopy A. De Rújula, H. Georgi, S.L. Glashow, Phys.

Rev. Lett. 38, 317 (1977)

[59] Quark Chemistry: Charmonium Molecules. A. De Rújula, R.L. Jaffe. Page 83 of EXPERIMEN-

TAL MESON SPECTROSCOPY 1977: Proceedings. Edited by Eberhard von Goeler and Roy

Weinstein. Boston, MA, Northeastern University Press, 1977

[60] W. Braunschweig et al., Phys. Lett. 57B, 407 (1975)

G.J. Feldman et al., Phys. Rev. Lett. 35, 821 (1975)

W. Tannenbaum et al., Phys. Rev. Lett. 35, 1323 (1975), 1125 (1975)

[61] J. Siegriest et al., Phys. Rev. Lett. 36, 701 (1976)

[62] A. De Rújula \& H. Georgi, Phys. Rev. D13, 1296 (1976)

[63] S.L. Adler, Phys. Rev. D10, 3714 (1974)

[64] E. Poggio, H. Quinn, \& S. Weinberg, Phys. Rev. D13, 1296 (1976)

[65] A. De Rújula, Charm is found, in Proceedings of the 1976 International Neutrino Conference, eds. H. Faissner, H. Reithler \& P. Zerwas, Aachen (Germany) 1976.

[66] G. Goldhaber in the same proceedings as reference [53].

[67] G. Goldhaber, F.M. Pierre et al., Phys. Rev. Lett. 37, 255 (1976)

[68] E. Eichten, K. Gottfried, T. Kinoshita, K.D. Lane \& Tung-Mow Yan, Phys. Rev. Lett. 36, 500 (1976)

E. Eichten \& K.D. Lane, Phys. Rev. Lett. 37, 477 (1976)

[69] J. Peruzzi et al., Phys. Rev. Lett. 37, 569 (1976)

[70] A. De Rújula, H. Georgi \& S.L. Glashow, Phys. Rev. Lett. 37, 398 (1976)

[71] The Seven Millennium Problems. The Clay Mathematics Institute of Cambridge, Massachusetts (CMI). http://claymath.org/millennium-problems/millennium-prize-problems

[72] H.D. Politzer, Phys. Lett. 70B, 430 (1977)

R.K. Ellis, H. Georgi, M. Machacek, H.D. Politzer \& G.G. Ross, Phys.Lett. 78B, 281 (1978) 